\title{
Galuteolin attenuates cerebral ischemia/reperfusion injury in rats via anti-apoptotic, anti-oxidant, and anti-inflammatory mechanisms
}

This article was published in the following Dove Press journal:

Neuropsychiatric Disease and Treatment

\author{
Xue Cheng' \\ Fan Zhang' \\ Jingwei $\mathrm{Li}^{2}$ \\ Gang Wang ${ }^{3}$ \\ 'Department of Neurology, The First \\ Affiliated Hospital of Jinzhou Medical \\ University, Jinzhou City, Liaoning \\ Province I2100I, People's Republic of \\ China; ${ }^{2}$ Department of Neurology, \\ Liaoning Health Industry Group Fuxin \\ Mine General Hospital, Jinzhou City, \\ Liaoning Province 121001 , People's \\ Republic of China; ${ }^{3}$ Department of \\ Interventional Radiology, The First \\ Affiliated Hospital of Jinzhou Medical \\ University, Jinzhou City, Liaoning \\ Province 12100I, People's Republic of \\ China
}

Purpose: Galuteolin is a substance extracted and purified from honeysuckle. The purpose of this study was to explore the protective effect of galuteolin on cerebral ischemia-reperfusion injury (CIRI) and reveal its potential molecular mechanism from the perspectives of antiapoptosis, anti-oxidation, and anti-inflammation.

Materials and methods: One hundred and fifty rats were randomly divided into five groups: sham group, ischemia-reperfusion (I/R) group, $50 \mathrm{mg} / \mathrm{kg}$ galuteolin group, $100 \mathrm{mg}$ / $\mathrm{kg}$ galuteolin group, and $200 \mathrm{mg} / \mathrm{kg}$ galuteolin group. Middle cerebral artery occlusion (MCAO) was used to establish a rat CIRI model, different doses of galtenolin were intraperitoneal injected $2 \mathrm{hrs}$ after ischemia, and then reperfusion was performed for 24 hrs. Neurological function and cerebral water content were determined, and cerebral infarct volume was evaluated by TTC staining. TUNEL staining was used to detect the apoptosis of nerve cells. Western Blot was used to detect the expressions of Akt, p-Akt, Sod1, Sod2, catalase, caspase-3, Bcl-2, and Bax. Lipid hydrogen peroxide (LPO) was determined by kit assay. The contents of vascular endothelial growth factor (VEGF) and pro-inflammatory cytokines IL- $1 \beta$ and TNF- $\alpha$ were determined by ELISA.

Results: The results showed that galuteolin could significantly reduce the cerebral infarction volume, neurologic score, and cerebral water content in a dose-dependent manner. In addition, galuteolin obviously reduced the apoptosis rate of nerve cells and the expression levels of caspase-3 and Bax, meanwhile up-regulated the expression levels of p-Akt and Bcl2. Furthermore, galuteolin apparently inhibited the levels of LPO, Sod1, Sod2, and catalase in the cerebral infarction tissues. Moreover, galuteolin also significantly reduced the levels of pro-inflammatory factors IL- $1 \beta$ and TNF- $\alpha$ in the cerebral infarction tissues. Finally, Galuteolin markedly inhibited the expression of VEGF in cerebral infarction tissues.

Conclusion: Galuteolin exerts neuroprotective effects against CIRI by inhibiting apoptosis, oxidation, and inflammation.

Keywords: galuteolin, ischemia, reperfusion, apoptosis, oxidation, inflammation

\section{Introduction}

Cerebral ischemia-reperfusion injury (CIRI) refers to the phenomenon that the blood flow reperfusion after a certain period of cerebral ischemia, which aggravates ischemic injury of brain tissue cells and is mainly characterized by nerve cell injury and apoptosis. ${ }^{1,2}$ Neuronal death caused by CIRI includes cell necrosis and apoptosis. ${ }^{3}$ Cell necrosis is an irreversible process of rapid and passive death. Apoptosis is a reversible active death process, which is the main way of neuron death after CIRI and is involved in the formation and development of nerve injury. ${ }^{4}$ Preventing apoptosis can
Correspondence: Gang Wang

Department of Interventional Radiology,

The First Affiliated Hospital of Jinzhou

Medical University, No. 2 Renmin Street, Jinzhou City, Liaoning Province 12100I,

People's Republic of China

Tel +864164197026

Email rwvmamsj05933442@I26.com 
reduce brain injury and the infarct volume after cerebral ischemia reperfusion. ${ }^{5}$ Neuronal apoptosis is a complex pathological process, mainly caused by neuronal degeneration, death, and loss of function, which result in neurological impairment symptoms. ${ }^{6}$ At present, studies have shown that the pathogenesis of nerve cell apoptosis induced by CIRI is not only related to the expression of apoptosis-related proteins, but also brain edema, calcium overload, inflammatory reaction, and oxidative stress. ${ }^{7,8}$ Cerebral edema is a typical symptom after cerebral ischemia reperfusion. ${ }^{9}$ Studies have found that cerebral ischemia reperfusion can induce a large number of oxygen free radicals, accelerate lipid peroxidation reaction, and acute inflammatory reaction, thus inducing apoptosis and aggravating the injury degree of ischemic brain tissue. ${ }^{10,11}$

Honeysuckle extract has anti-oxidant, anti-viral, and antiinflammatory effects, in which galuteolin is one of the main active components. ${ }^{12}$ Galuteolin is a natural flavonoid, which has many pharmacological activities such as anti-oxidation, anti-infection, anti-tumor, and anti-inflammation. ${ }^{13}$ Galutelin was found to have different therapeutic effects on the central nervous system, cardiovascular system, and respiratory system diseases. ${ }^{14,15}$ Fan et al, found that galuteolin could inhibit the proliferation and metastasis of hepatocellular carcinoma cells, and the main mechanism was to inhibit the expression of NLRP3 inflammatory bodies in hepatocellular carcinoma cells. ${ }^{16}$ Wang et al, showed that galuteolin could block the activation of NF- $\mathrm{kB}$ and TLR2 signaling pathways, thereby reducing the inflammation, injury and apoptosis of uterine cells caused by staphylococcus aureus. ${ }^{17}$ Liu et al, reported that galuteolin could reduce myocardial cell injury caused by myocardial ischemia reperfusion by reducing oxidative stress response and inhibiting myocardial cell apoptosis. ${ }^{18}$ However, there are no study on the effect of galuteolin on CIRI. In this study, a rat model of cerebral ischemia was established using the modified suture method, and the model of CIRI in rats was successfully constructed by reperfusion for $24 \mathrm{hrs}$ after $2 \mathrm{hrs}$ of ischemia. The aim of this study was to investigate the protective mechanism of galuteolin against CIRI in vivo.

\section{Materials and methods}

\section{Animals}

One hundred and fifty healthy male SD rats weighing 200 $220 \mathrm{~g}$ were purchased from the experimental animal center of The First Affiliated Hospital of Jinzhou Medical University. The rats were kept in a $12 \mathrm{hr}$ light-dark cycle and fed freely.
All experiments were conducted in accordance with the national institutes of health laboratory animal care and use guidelines, and were approved by The First Affiliated Hospital of Jinzhou Medical University animal care and use committee.

\section{Establishment of CIRI model}

The CIRI ( $2 / 24 \mathrm{hrs})$ model was established by a modified MCAO methods. In the whole process, the temperature of rats was maintained at $37^{\circ} \mathrm{C}$. Firstly, rats were anesthetized with $10 \%(\mathrm{w} / \mathrm{v})$ chloral hydrate $(350 \mathrm{mg} / \mathrm{kg}$ body weight) by intraperitoneal injection. Then, the left common carotid artery (CCA) was exposed and the external carotid artery (ECA) was isolated. Hemostatic clips were used to block CCA and ECA. Next, the tip of the heparin coating with a diameter of $0.25-0.28 \mathrm{~mm}$ nylon monofilament was inserted from the CCA into the internal carotid artery (ICA) to prevent the origin of the middle cerebral artery. Local cerebral blood flow (rCBF) was measured using a laser doppler flowmeter, and when $\mathrm{rCBF}$ decreased below $20 \%$ of the baseline, arterial occlusion was successful. After MCAO for $2 \mathrm{hrs,} \mathrm{nylon} \mathrm{monofilaments} \mathrm{were}$ removed and keep reperfusion for $24 \mathrm{hrs}$. The rats in the sham group were treated with MCAO, but the nylon monofilament was not inserted into CCA.

\section{Grouping and administration}

The galuteolin used in the experiment was purchased from Sigma with purity greater than $98 \%$. The chemical structure of galuteolin was shown in Figure 1A. One hundred and fifty rats were randomly divided into five groups: sham group, I/R group, $50 \mathrm{mg} / \mathrm{kg}$ galuteolin group, 100 $\mathrm{mg} / \mathrm{kg}$ galuteolin group, and $200 \mathrm{mg} / \mathrm{kg}$ galuteolin group, with 30 rats in each group. Two hours after ischemia, the rats in the galtenolin treatment group were injected with different doses of galtenolin peritoneally, while the sham group and I/R group were simultaneously injected with the same volume of normal saline. The rats were anesthetized and sacrificed $24 \mathrm{hrs}$ after reperfusion. The experimental process was shown in Figure 1B.

\section{TTC staining}

TTC staining was used to determine the volume of cerebral infarction in rats. The rats in each group $(n=6)$ were anesthetized and sacrificed. Brain tissue was taken and cut into $1.5 \mathrm{~mm}$ thick slices which were stained with $1 \%$ TTC for $0.5 \mathrm{hrs}$ and fixed with $4 \%$ paraformaldehyde. TTC could only stain normal tissue red, while infarct tissue 
A<smiles>O=c1cc(-c2ccc(O)c(O)c2)oc2cc(O[C@@H]3O[C@H](CO)[C@@H](O)[C@H](O)[C@H]3O)cc(O)c12</smiles>

B

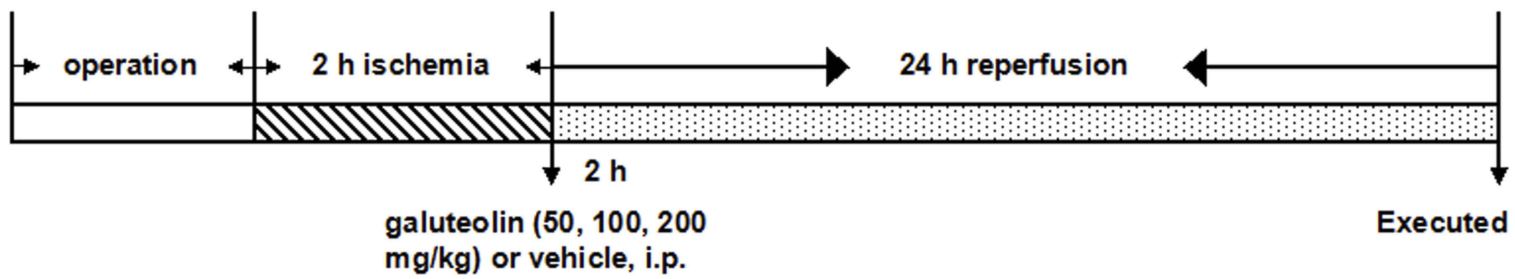

Figure I The chemical structure of galuteolin and the experimental process. (A) Schematic diagram of the chemical structure of galuteolin $\left(\mathrm{C}_{21} \mathrm{H}_{20} \mathrm{O}_{1 \mid}\right.$, molecular weight: 448.38). (B) Flow chart of the establishment of rat MCAO/R model.

would not be stained and keep white. Brain tissue sections were photographed, and the volume of the sections was quantified using Image $J$ software. The percentage of infarcts was the ratio of infarct volume to total volume.

\section{Neurological score}

Neurological score was used to evaluate the effect of galuteolin on I/R-induced nerve injury in rats. The neurological behaviors of rats in each group $(n=6)$ were observed and scored after reperfusion for $24 \mathrm{hrs}$. The specific scoring criteria were: (1) normal performance, 0 points; (2) the left forelegs of the rats could not be fully extended, 1 point; (3) turn the rat, 2 points; (4) the rats fell to the left side; (5) the rats lost consciousness, 4 points.

\section{Determination of cerebral water content} Cerebral water content of rat in each group $(n=6)$ was determined after reperfusion for $24 \mathrm{hrs}$. First, the wet weight of infarct cerebral hemisphere in rats was measured. Then, the brain tissue was dried under $105{ }^{\circ} \mathrm{C}$, and the dry weight of infarction cerebral hemisphere was measured. Total brain water: [(wet weight-dry weight)/ wet weight] $\times 100 \%$.

\section{TUNEL staining}

TUNEL apoptosis assay kit (Beyotime) was used to detect the apoptosis of brain neurons in each group $(n=6)$. Brain sections were stained and photographed under an optical microscope. The apoptosis rate of neurons was the percentage of TUNEL positive cells in the total number of neurons.

\section{ELISA assay}

The expression levels of TNF- $\alpha$, IL-1 $\beta$, and VEGF in cerebral infarction tissues were determined by ELISA. In short, the infarcted tissue in rats in PBS buffer containing protease inhibitors was homogenized and centrifuged to obtain the supernatant. The expression levels of TNF- $\alpha$, IL- $1 \beta$, and VEGF in cerebral infarction tissues were determined by ELISA, and the specific procedures were strictly in accordance with the instructions of the kit.

\section{Determination of LPO}

The LPO assay kit (Cayman Chemical) was used to determine the content of LPO in the cerebral infarction tissues of rats, so as to evaluate the level of lipid peroxidation in the tissues. The content of LPO was expressed in $\mu \mathrm{M}$ of LPO per milligram of protein.

\section{Western blot assay}

The total proteins extracted from cerebral infarction were separated by $10 \%$ SDS-PAGE separation gel. And then the separated proteins were transferred to cellulose acetate membrane. Using PBS solution containing 5\% dried skim milk closed for $1 \mathrm{hr}$, and then incubated with the primary antibody at $4^{\circ} \mathrm{C}$ for the night. Bcl-2, Bax, caspase3, Sod1, Sod 2, catalase, Akt, p-Akt, and $\beta$-actin were obtained from Santa Cruz Biotechnology. Next, the secondary antibody was used to incubate the membrane at room temperature for $1 \mathrm{hr}$, and the protein bands were stained with ECL chemiluminescence solution. 


\section{Statistical analysis}

Statistical analysis was performed using the SPSS 17.0 software. The results represent the mean of three independent experiments, and the data are presented as the means \pm standard deviation (SD). Comparisons between experimental and control groups were performed by one-way analysis of variance (ANOVA) followed by Tukey test. Differences were considered significant when $P<0.05$.

\section{Results}

\section{Effects of galuteolin on cerebral infarction volume, neural behavior, and cerebral water content after CIRI in rats}

In this study, TTC staining was used to evaluate the effect of galuteolin on cerebral infarction volume after CIRI in rats. The results showed that the cerebral infarction volume in the $\mathrm{I} / \mathrm{R}$ group was significantly higher than that in the sham group $(P<0.01)$, while the cerebral infarction volume in the galuteolin treated groups was remarkably lower than that in the $\mathrm{I} / \mathrm{R}$ group, with the highest inhibition rate of cerebral infarction reaching $62.33 \%$ (Figure $2 \mathrm{~A}$ and $\mathrm{B}, P<0.01$ ). In addition, we evaluated the neurological status of rats. The results showed that the neurologic score of the $\mathrm{I} / \mathrm{R}$ group was apparently higher than that of the sham group $(P<0.01)$, while the neurologic score of the galuteolin treated groups was lower than that of the $\mathrm{I} / \mathrm{R}$ group (Figure $2 \mathrm{C}, P<0.05$, $P<0.01)$. Furthermore, the results of the content of brain water in rats showed that $I / R$ significantly increased the cerebral water content of the rats $(P<0.01)$, while the cerebral water content of the rats treated with different doses of galuteolin was lower than that of the $\mathrm{I} / \mathrm{R}$ group (Figure 2D, $P<0.05, P<0.01)$. The above results indicated that different doses of galuteolin could alleviate the brain damage caused by CIRI in rats in a dose-dependent manner.
A
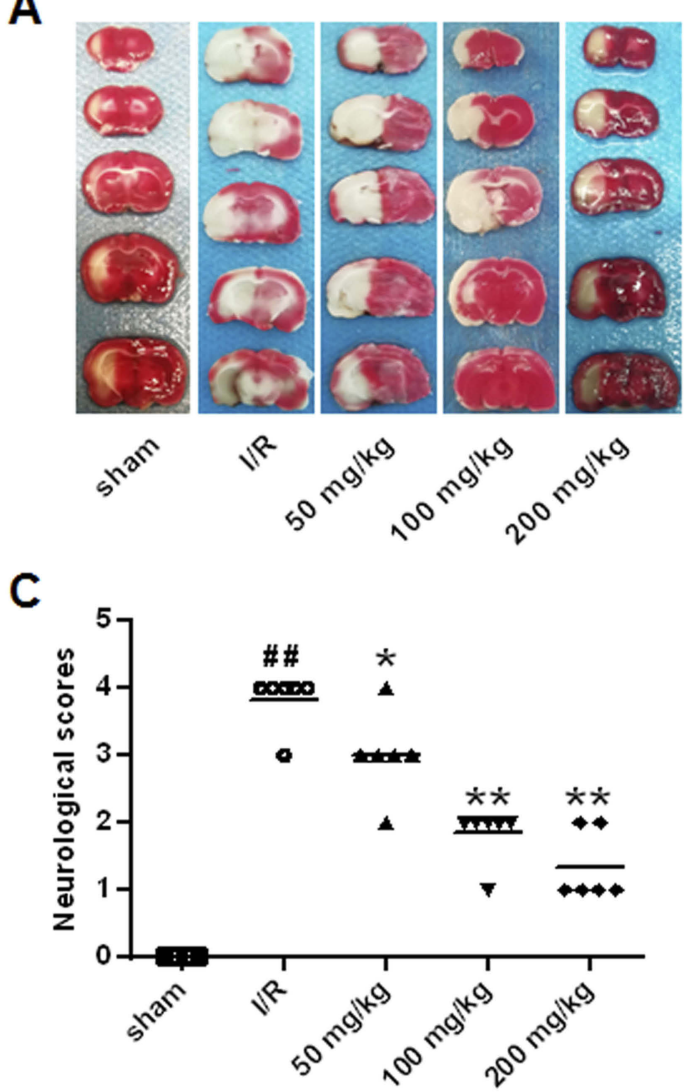

B

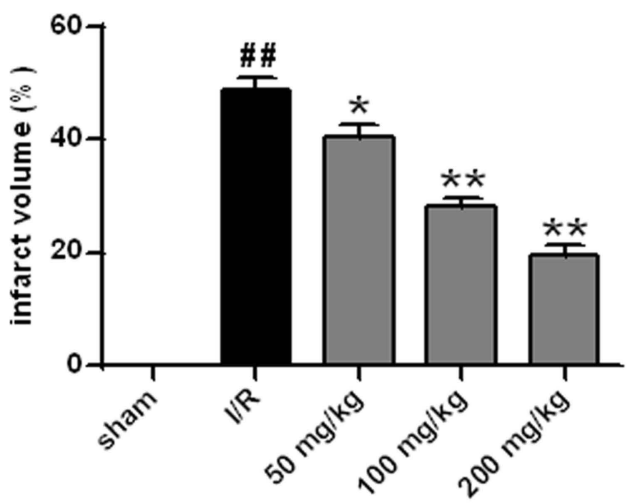

D

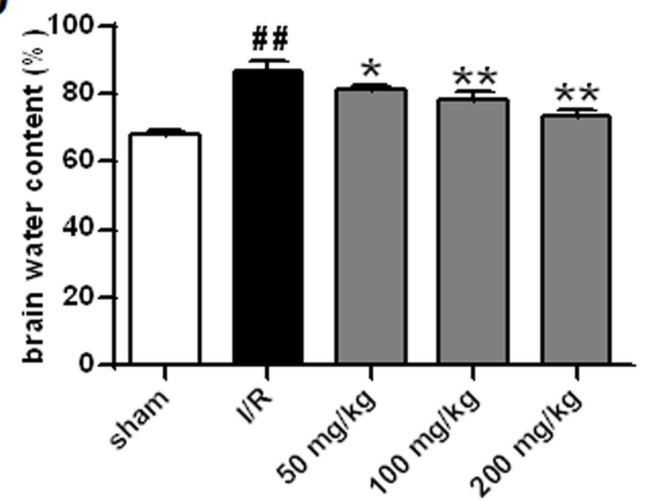

Figure 2 Effects of galuteolin on cerebral infarction volume, neural behavior, and cerebral water content after CIRI in rats. Effects of galuteolin on cerebral infarction volume $(\mathbf{A}, \mathbf{B})$, neural behavior, $(\mathbf{C})$ and cerebral water content, and (D) after MCAO/R injury in rats.

Notes: $n=6$, compared with the I/R group $p,{ }^{*} p<0.05$, ${ }^{* * P}<<0.01$; compared with the sham group, ${ }^{\#} p<0.01$. 


\section{Effects of galuteolin on apoptosis of nerve cells and apoptosis-related proteins in rat cerebral infarction}

The function of galuteolin in the apoptosis of nerve cells in rat cerebral infarction was determined by TUNEL staining. The results showed that the percentage of TUNEL positive cells in the I/R group was significantly higher than that in the sham group $(P<0.01)$, while the percentage of TUNEL positive cells in the galtenolin treatment groups were remarkably lower than that in the $\mathrm{I} / \mathrm{R}$ group (Figure $3 \mathrm{~A}$ and $\mathrm{B}, P<0.05$, $P<0.01$ ), which indicated that galuteolin could reduce the mortality of nerve cells in the cerebral infarction tissue of rats.

The effect of galuteolin on the expressions of Bcl-2, Bax, and caspase- 3 in cerebral infarction tissues was determined by Western Blot. The results showed that, compared with the sham group, the protein expression of Bcl-2 was decreased in the I/R group (Figure $4 \mathrm{~A}$ and $\mathrm{B}, P<0.01$ ), while the expressions of Bax and caspase- 3 were up-regulated (Figure $4 \mathrm{~A}, \mathrm{C}$ and $\mathrm{D}, P<0.05, P<0.01)$. The protein expression of $\mathrm{Bcl}-2$ in galbutolin treatment groups was remarkably higher than that in $\mathrm{I} / \mathrm{R}$ group (Figure $4 \mathrm{~A}$ and $\mathrm{B}, P<0.05, P<0.01$ ), while the protein levels of Bax and caspase- 3 were lower than that in $\mathrm{I} / \mathrm{R}$ group (Figure $4 \mathrm{~A}, \mathrm{C}$ and $\mathrm{D}, P<0.05, P<0.01$ ), and the ratio of Bcl-2 to Bax was significantly higher than that in I/R group (data not shown). These results indicate that galuteolin could inhibit I/R-induced apoptosis by regulating the expression of apoptosis-related proteins. Study found that activation of Akt signaling pathway can inhibit the apoptosis of cells. ${ }^{19}$ In our research, Western Blot was used to determine the effect of galuteolin on Akt in cerebral infarction. The results showed that, compared with the sham group, the expression of $\mathrm{p}$-Akt was markedly decreased in the I/R group (Figure $5 \mathrm{~A}$ and $\mathrm{B}, P<0.01$ ). The protein level of $\mathrm{p}$-Akt in galbutolin treatment groups was remarkably higher than that in $\mathrm{I} / \mathrm{R}$ group (Figure $5 \mathrm{~A}$ and $\mathrm{B}, P<0.01$ ), indicating that galuteolin could inhibit the I/R-induced apoptosis in cerebral infarction tissue by activating Akt.

\section{Effect of galuteolin on oxidative stress in cerebral infarction of rats}

Oxidative stress is the main manifestation of ischemic brain injury. ${ }^{20}$ In this study, the effect of galuteolin on the level of oxidative stress was evaluated by measuring the level of LPO in the cerebral infarction of rats. Compared with the sham group, the level of LPO in the I/R group was significantly higher (Figure 6A, $P<0.01$ ), while the level of LPO in the galuteolin treatment groups was significantly lower than that in the I/R group (Figure $6 \mathrm{~A}, P<0.05, P<0.01$ ). In addition, anti-oxidant activity is a relative factor to reflect

A

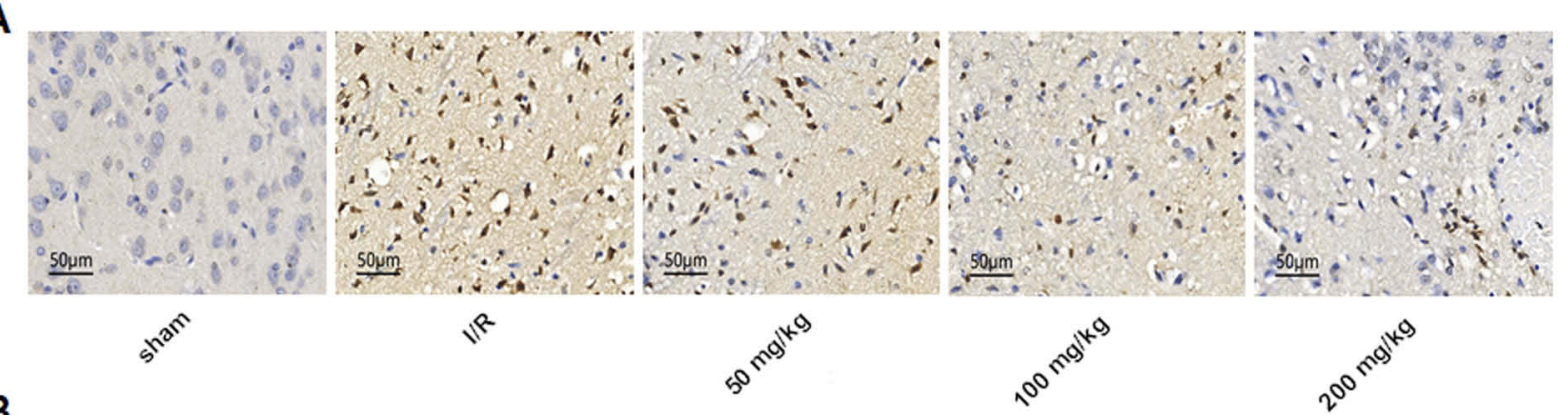

B

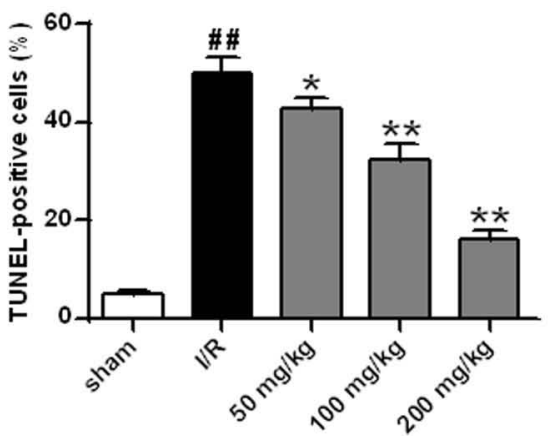

Figure 3 Detection of the effect of galuteolin on neuronal apoptosis by TUNEL staining. (A) Images of TUNEL staining in rat brain tissue from each group. (B) TUNEL positive cells in the rat brain tissue of each group.

Notes: $n=6$, compared with the I/R group $p, * P<0.05$, **P<0.0I; compared with the sham group, ${ }^{\#} P<0.01$. 


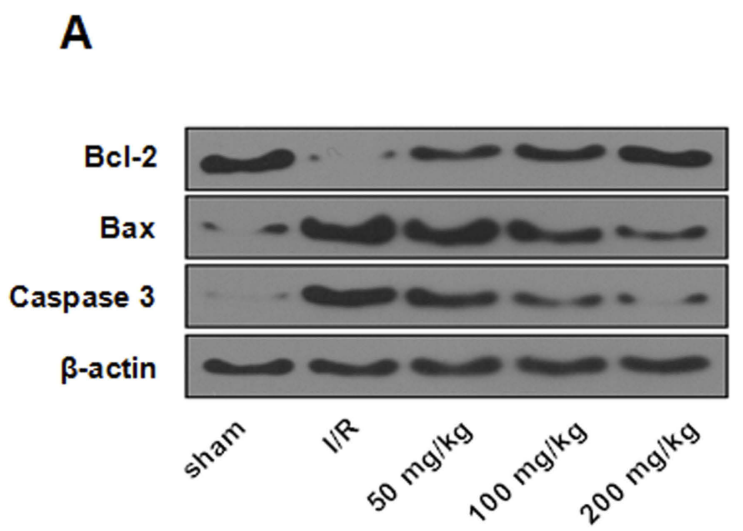

B

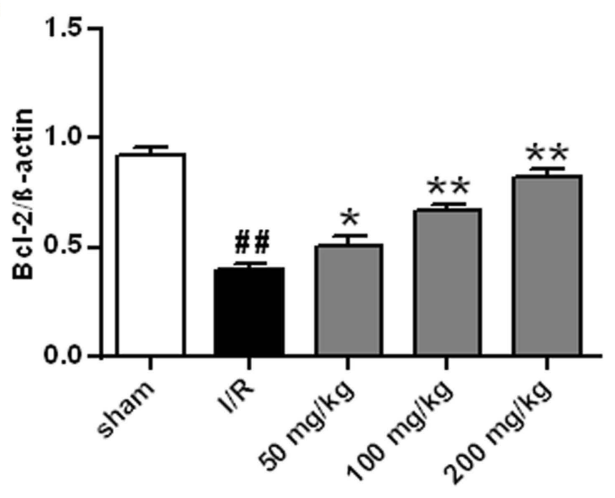

C

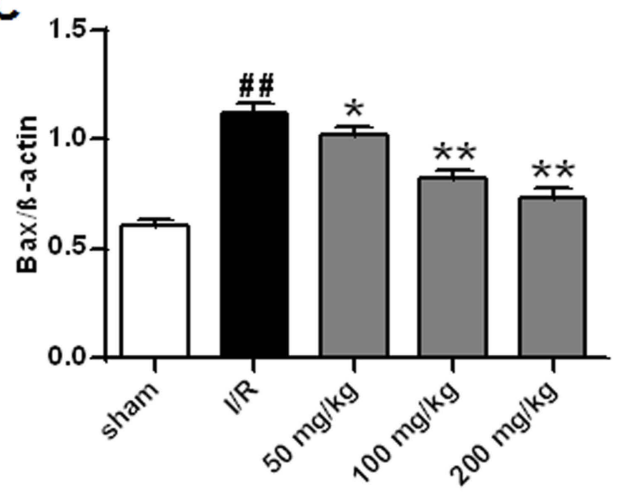

D

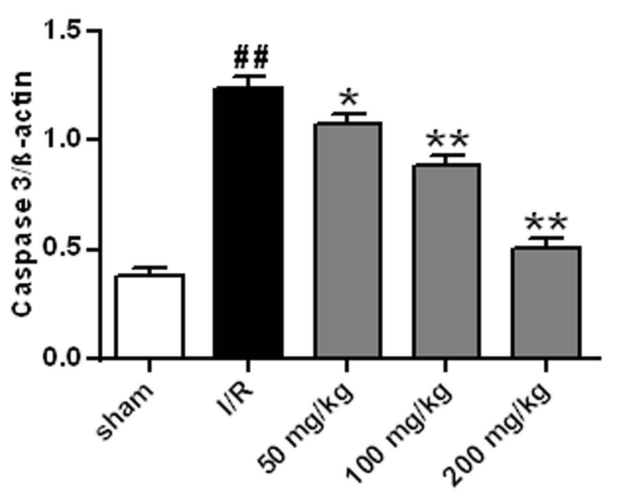

Figure 4 Effects of galuteolin on apoptosis-related proteins in rat cerebral infarction tissues. (A) Determination of the protein expressions of Bcl-2, Bax, and caspase-3 in cerebral infarction tissues of rats by Western Blot. (B) Histogram of BCL-2 protein expression. (C) Histogram of protein expression of Bax. (D) Histogram of caspase-3 protein expression. The $\boldsymbol{\beta}$-actin as a standard internal reference.

Notes: $n=6$, compared with the I/R group $p, * P<0.05$, $* * P<0.01$; compared with the sham group, ${ }^{\prime} P<0.01$.
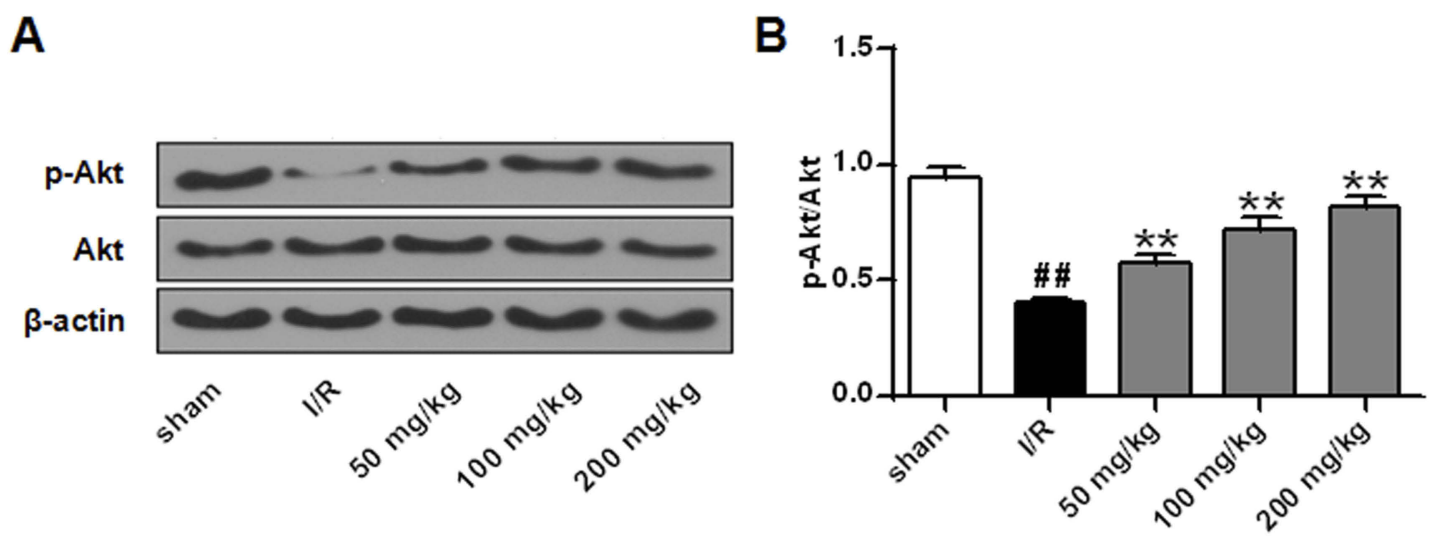

Figure 5 Effects of galuteolin on Akt phosphorylation in rat cerebral infarction tissues. (A) Determination of the expressions of p-Akt and Akt in rat cerebral infarction by Western Blot. (B) Histogram of p-AktAkt protein expressions. The $\boldsymbol{\beta}$-actin as a standard internal reference.

Notes: $\mathrm{n}=6$, compared with the I/R group, ${ }^{* * P}<0.0$ I; compared with the sham group, ${ }^{\#} P<0.01$.

the level of oxidative stress; therefore, the protein expression levels of Sod1, Sod2, and catalase in cerebral infarction tissues of rats were determined by Western Blot. The results showed that the protein levels of Sod1, Sod2, and catalase in the cerebral infarction tissues of rats in the $\mathrm{I} / \mathrm{R}$ group were lower than those in the sham group (Figure 6B-E, $P<0.01$ ), while the protein levels of Sod1, Sod2, and catalase in the $100 \mathrm{mg} / \mathrm{kg}$ and $200 \mathrm{mg} / \mathrm{kg}$ galuteolin treatment groups were remarkably up-regulated, compared with the $\mathrm{I} / \mathrm{R}$ group (Figure $6 \mathrm{~B}-\mathrm{E}, P<0.01$ ). The above results indicated that 
A

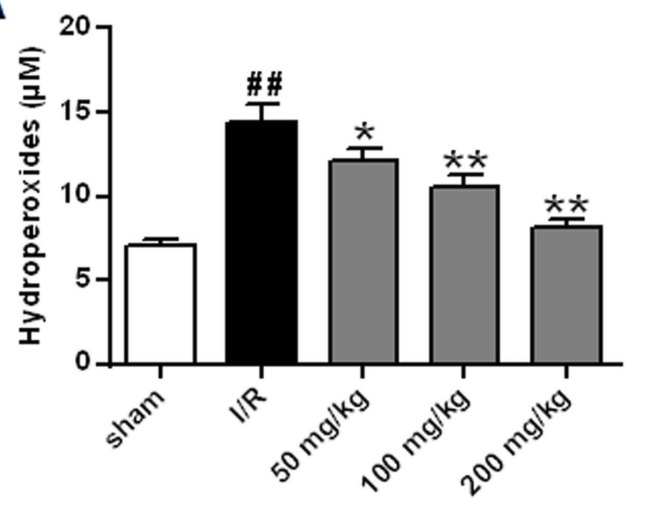

C

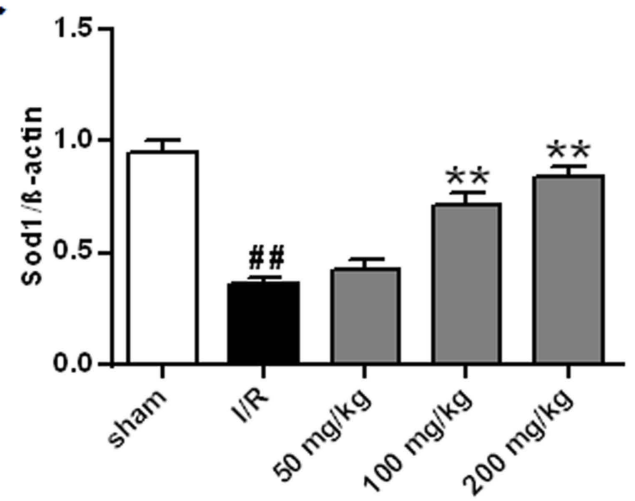

E

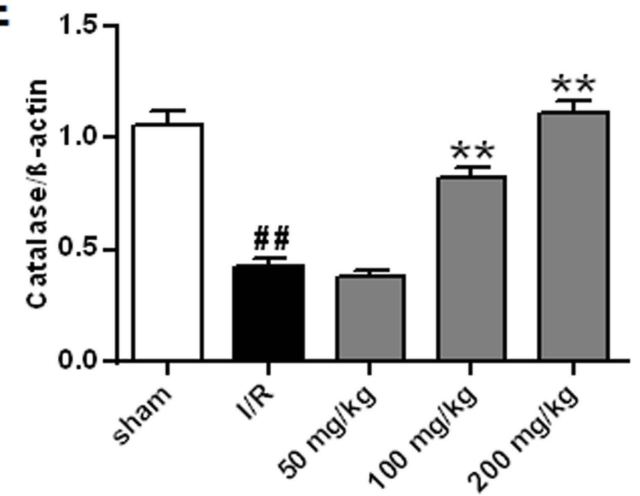

B

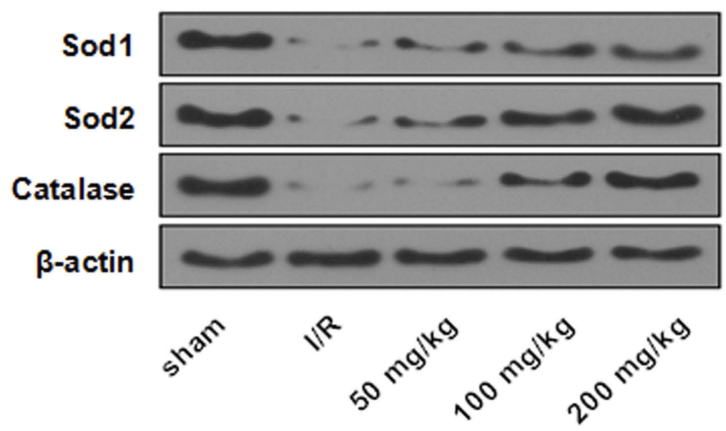

D

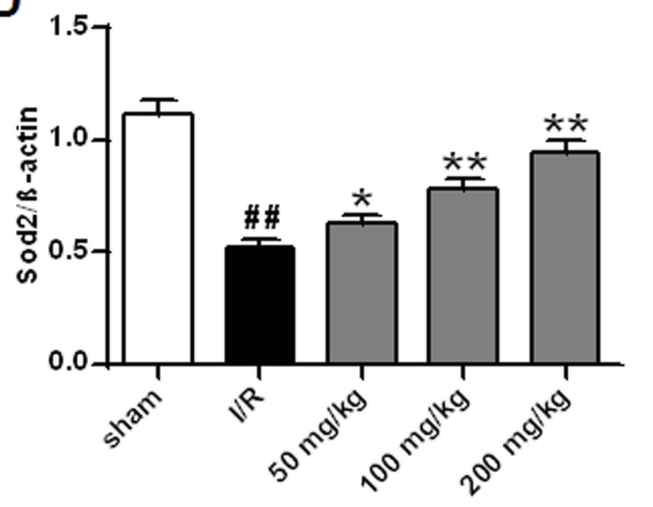

Figure 6 Effects of galuteolin on oxidative stress in cerebral infarction of rats. (A) Determination of the level of LPO in rat brain tissue by LPO kit. (B) Determination of the expression levels of SodI, Sod2, and catalase by Western Blot. (C) Histogram of Sodl protein expression. (D) Histogram of Sod2 protein expression. (E) Histogram of catalase protein expression. The $\boldsymbol{\beta}$-actin as a standard internal reference.

Notes: $n=6$, compared with the I/R group $p, * P<0.05$, $* * P<0.01$; compared with the sham group, ${ }^{\#} P<0.01$

galuteolin could reduce the oxidative stress level in the cerebral infarction tissue caused by $\mathrm{I} / \mathrm{R}$.

\section{Effect of galuteolin on inflammatory responses in rat cerebral infarction}

The secretion of pro-inflammatory cytokines TNF- $\alpha$ and IL$1 \beta$ in cerebral infarction was determined by ELISA. The results showed that the secretions of TNF- $\alpha$ and IL- $1 \beta$ in the cerebral infarction tissues of the I/R group were significantly higher than those in the sham group (Figure 7A and B,
$P<0.01$ ), while the secretions of TNF- $\alpha$ and IL- $1 \beta$ in the galuteolin treatment groups were decreased, compared with the $\mathrm{I} / \mathrm{R}$ group (Figure $7 \mathrm{~A}$ and $\mathrm{B}, P<0.05, P<0.01$ ), which indicated that galuteolin could reduce the inflammatory response in the cerebral infarction tissue caused by I/R.

\section{Effect of galuteolin on VEGF level in cerebral infarction tissue of rats}

Neovascularization may be a natural defense mechanism to recover the nutrition supply to brain tissue after $I / R$ 
A

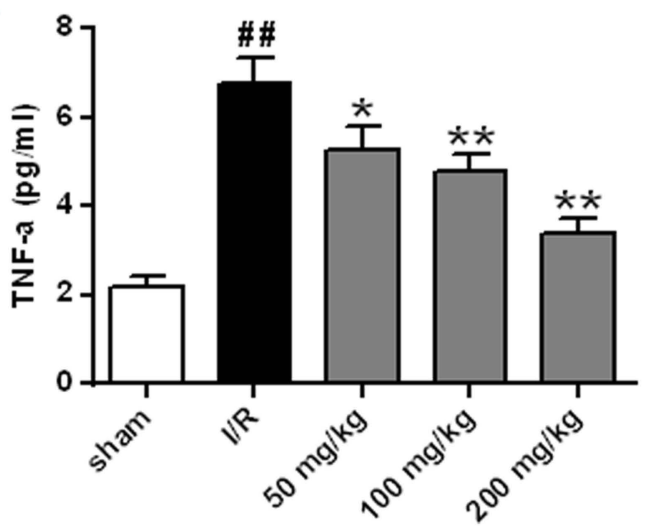

B

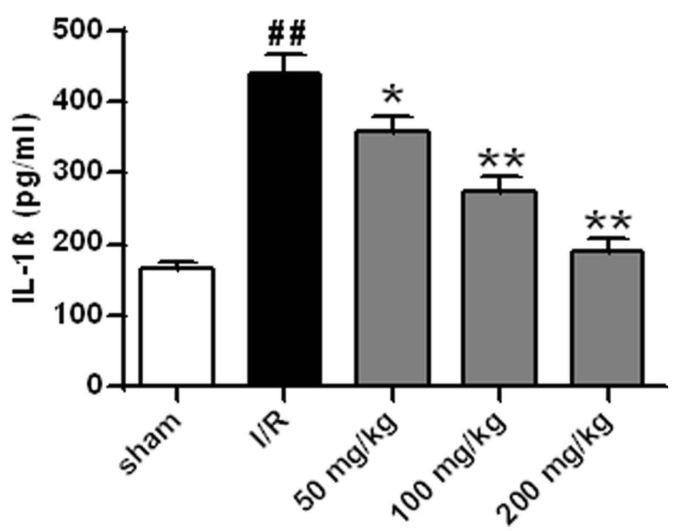

Figure 7 Effects of galuteolin on inflammatory responses in rat cerebral infarction. (A) Determination of the secretion of pro-inflammatory cytokine TNF- $\boldsymbol{\alpha}$ in cerebral infarction by ELISA. (B) Determination of the secretion of pro-inflammatory cytokine IL- I $\beta$ in cerebral infarction by ELISA.

Notes: $n=6$, compared with the I/R group $p$, $* P<0.05$, $* * P<0.01$; compared with the sham group, $P<0.01$.

injury in the human body. VEGF is a factor that promotes the growth of vascular endothelial cells. ${ }^{21}$ The effect of galuteolin on VEGF level in rat cerebral infarction was determined by ELISA. Compared with the sham group, the results showed that the level of VEGF in the I/R group was increased (Figure $8, P<0.01$ ), while the level of VEGF in the $100 \mathrm{mg} / \mathrm{kg}$ and $200 \mathrm{mg} / \mathrm{kg}$ galuteolin treatment groups was significantly lower than that in the $\mathrm{I} / \mathrm{R}$ group (Figure $8, P<0.01$ ). It was speculated that galuteolin might inhibit new angiogenesis in the cerebral infarction tissue caused by $\mathrm{I} / \mathrm{R}$ through reducing the level of VEGF in the cerebral infarction tissue of rats.

\section{Discussion}

Acute cerebral ischemia is a common cerebrovascular disease with high morbidity and disability. ${ }^{22}$ Studies have found that reperfusion of the ischemic site can aggravate brain tissue injury, and the main injury mechanism is related to apoptosis of nerve cells, inflammatory reaction, oxidative stress injury, etc. ${ }^{23}$ Zhan et al, found that dendrobium polysaccharide could reduce the damage caused by focal cerebral ischemia reperfusion by reducing the cerebral infarction volume, cerebral water content, and score of neural function defect in rats. ${ }^{24}$ Huang et al, reported that hydrogen inhalation improved the degree of neural function injury in MCAO mice after cerebral $\mathrm{I} / \mathrm{R}$ by reducing the score of neural behavior defect, cerebral water content, and cerebral infarction volume. ${ }^{25}$ In this study, we first investigated the effects of galuteolin on CIRI and revealed its potential molecular mechanism in alleviating the CIRI of rats. Results showed that galuteolin

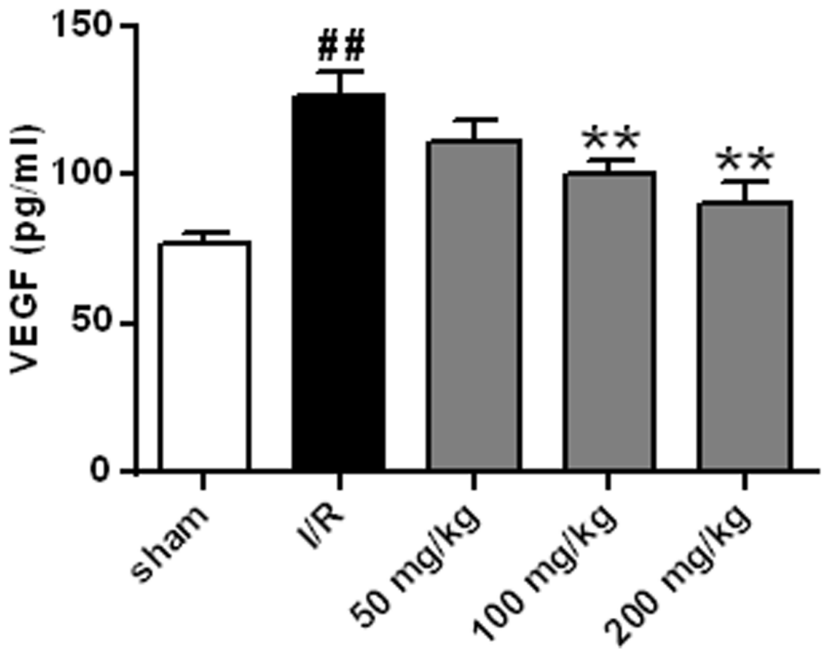

Figure 8 Effect of galuteolin on VEGF level in cerebral infarction tissue of rats. Notes: $n=6$, compared with the I/R group $p, * * p<0.01$; compared with the sham group, ${ }^{\#} \mathrm{P}<0.01$.

could reduce the CIRI by decreasing the cerebral infarction volume and neurological scoring, brain water content.

Nerve cell injury and apoptosis are the main pathological features of CIRI, which are closely related to the expression of apoptosis-related proteins. ${ }^{26} \mathrm{Bcl}-2$ is an antiapoptotic gene, while Bax is a pro-apoptotic gene, and the ratio of Bcl-2/Bax can reflect the survival status of cells. ${ }^{27}$ Caspase- 3 is the terminal protein of the cell apoptosis protein cascade reaction, which can promote the cell apoptosis. ${ }^{28} \mathrm{PI} 3 \mathrm{~K} / \mathrm{Akt}$ is a classical anti-apoptotic signaling pathway, which can up-regulate the phosphorylation level of Akt after activation, thereby activating the antiapoptotic mechanism of cells and inhibiting apoptosis. ${ }^{29}$ Sun et al, found that galuteolin could significantly reduce 
myocardial infarction area and apoptosis rate of myocardial cells, reduce the release of lactic dehydrogenase $(\mathrm{LDH})$, up-regulate the expression of anti-apoptotic protein Bcl-2, activate the PI3K/Akt pathway, and thereby improve cardiac function after myocardial ischemia/reperfusion injury in rats. ${ }^{30}$ In this study, the results indicated that galuteolin could reduce the death rate of nerve cells in the cerebral infarction tissue of rats in some pathways: (1) up-regulate the protein levels of Bcl-2 and down-regulate the expressions of Bax. (2) Up-regulate the protein levels of p-Akt to activate the PI3K/Akt anti-apoptotic signaling pathway. (3) Down-regulate the expressions of caspase-3 to inhibit cell apoptosis.

Currently, studies have suggested that the pathogenesis of nerve cell apoptosis induced by CIRI is related to cerebral edema, calcium overload, inflammatory reaction, and oxidative stress. ${ }^{31}$ LPO is the product of the reaction between oxygen free radicals and polyunsaturated fatty acids. ${ }^{32}$ Sod is an enzyme that scavenges superoxide anion free radicals in vivo, which can effectively relieve lipid peroxidation caused by free radicals. ${ }^{33}$ Catalase can catalyze the decomposition of hydrogen peroxide into oxygen and water, which has antioxidant activity. ${ }^{34}$ In this study, we found that galuteolin could reduce the level of oxidative stress in the cerebral infarction tissue caused by $\mathrm{I} / \mathrm{R}$ through decreasing the reaction between oxygen free radicals and polyunsaturated fatty acids, meanwhile, activating the production of Sod1, Sod2 to relieve lipid peroxidation caused by free radicals.

Inflammatory response is one of the important pathological mechanisms that aggravate CIRI. Activated white blood cells and endothelial cells after injury will secrete a large amount of pro-inflammatory factors such as IL-1 $\beta$ and TNF- $\alpha$, thus causing inflammatory response and aggravating the degree of brain injury. ${ }^{35}$ The results of this study indicated that galuteolin could inhibit the secretion levels of TNF- $\alpha$ and IL-1 $\beta$. Study has found that luteolin which is also a natural flavonoid like galuteolin can inhibit TNF- $\alpha$ and NF- $\kappa$ B pathway by binding to TNFR1, TRADD, TRAF2, and RIP1 through covalent bonding and blocking the ATP binding sites of IKK $\hat{I}^{2.36}$ Therefore, we speculated that galuteolin could also to reduce the inflammatory response by binding to some signal molecules or blocking some combinations, which needed further investigation.

\section{Conclusion}

In conclusion, the present study assessed the protective effect of galuteolin on CIRI and its potential molecular mechanism. The results indicated that galuteolin could play a neuroprotective role against CIRI by inhibiting apoptosis, oxidation, and inflammation, which would provide certain theoretical basis for the treatment of CIRI with galuteolin.

\section{Ethical approval}

All experiments were conducted in accordance with the National Institutes of Health Laboratory Animal Care and Use Guidelines, and were approved by the Animal Care and Use Committee of The First Affiliated Hospital of Jinzhou Medical University.

\section{Acknowledgment}

This work was supported by the Liaoning Natural Science Foundation (201602273).

\section{Disclosure}

The authors report no conflicts of interest in this work.

\section{References}

1. Sun J, Wang F, Ling Z, et al. Clostridium butyricum attenuates cerebral ischemia/reperfusion injury in diabetic mice via modulation of gut microbiota. Brain Res. 2016;1642:180-188. doi:10.1016/j.brainres.2016.03. 042

2. Ma Y, Lu C, Li C, et al. Overexpression of HSPA12B protects against cerebral ischemia/reperfusion injury via a PI3K/Akt-dependent mechanism. Biochim Biophys Acta. 2013;1832(1):57-66. doi:10.10 16/j.bbadis.2012.10.003

3. Zhang Y, Lin R, Tao J, et al. Electroacupuncture improves cognitive ability following cerebral ischemia reperfusion injury via CaMCaMKIV-CREB signaling in the rat hippocampus. Exp Ther Med. 2016;12(2):777-782. doi:10.3892/etm.2016.3428

4. Gong J, Sun F, Li Y, et al. Momordica charantia polysaccharides could protect against cerebral ischemia/reperfusion injury through inhibiting oxidative stress mediated c-Jun N-terminal kinase 3 signaling pathway. Neuropharmacology. 2015;91:123-134. doi:10.1016/j.neuropharm.2014. 11.020

5. Zhao S, Yin J, Zhou L, et al. Hippo/MST1 signaling mediates microglial activation following acute cerebral ischemia-reperfusion injury. Brain Behav Immun. 2016;55:236-248. doi:10.1016/j.bbi.2015.12. 016

6. Yun Q, Jiang M, Wang J, et al. Overexpression Bax interacting factor1 protects cortical neurons against cerebral ischemia-reperfusion injury through regulation of ERK1/2 pathway. J Neurol Sci. 2015;357 (1):183-191. doi:10.1016/j.jns.2015.07.027

7. Chen B, Wu Z, Xu J, Xu Y. Calreticulin binds to fas ligand and inhibits neuronal cell apoptosis induced by ischemia-reperfusion injury. Biomed Res Int. 2015;2015:895284. doi:10.1155/2015/895284

8. Liao J, Ye Z, Huang G, Xu C, Guo Q, Wang E. Delayed treatment with NSC23766 in streptozotocin-induced diabetic rats ameliorates postischemic neuronal apoptosis through suppression of mitochondrial p53 translocation. Neuropharmacology. 2014;85:508-516. doi:10.10 16/j.neuropharm.2014.06.008

9. Ma L, Xing G, Yu Y, et al. Sulforaphane exerts neuroprotective effects via suppression of the inflammatory response in a rat model of focal cerebral ischemia. Int J Clin Exp Med. 2015;8(10):17811-17817. 
10. Jiang M, Li J, Peng Q, et al. Neuroprotective effects of bilobalide on cerebral ischemia and reperfusion injury are associated with inhibition of pro-inflammatory mediator production and down-regulation of JNK1/2 and p38 MAPK activation. J Neuroinflammation. 2014;11 (1):167. doi:10.1186/s12974-014-0167-6

11. Han D, Zhang S, Fan B, et al. Ischemic postconditioning protects the neurovascular unit after focal cerebral ischemia/reperfusion injury. $J$ Mol Neurosci. 2014;53(1):50-58. doi:10.1007/s12031-013-0196-0

12. Xin H, Feng J, Cheng R-M, Xin N. Simultaneous determination of chlorogenic acid and galuteolin in honeysuckle. Chin J Exp Tradit Med Formulae. 2011;2.

13. Zhang X, Tong J, Zhou Y, Xiaoyu X. Studies on medicinal ingredients and pharmacological effects of Lonicera. Chin Pharmacol Bull. 2014;8:1049-1054.

14. Wang G, Li W, Lu X, Bao P, Zhao X. Luteolin ameliorates cardiac failure in type I diabetic cardiomyopathy. J Diabetes Complications. 2012;26(4):259-265. doi:10.1016/j.jdiacomp.2012.04.007

15. Lv L, Lv L, Zhang Y, Kong Q. Luteolin prevents LPS-induced TNF$\alpha$ expression in cardiac myocytes through inhibiting NF- $\mathrm{NB}$ signaling pathway. Inflammation. 2011;34(6):620-629. doi:10.1007/s10753010-9271-7

16. Fan S, Wang Y, Lu J, et al. Luteoloside suppresses proliferation and metastasis of hepatocellular carcinoma cells by inhibition of NLRP3 inflammasome. PLoS One. 2014;9(2):e89961.

17. Wang X, Yuan T, Yin N, et al. Luteoloside protects the uterus from staphylococcus aureus-induced inflammation, apoptosis, and injury. Inflammation. 2018;41(5):1702-1716. doi:10.1007/s10753-018-0814-7

18. Liu Z, Yang L, Huang J, et al. Luteoloside attenuates anoxia/reoxygenation-induced cardiomyocytes injury via mitochondrial pathway

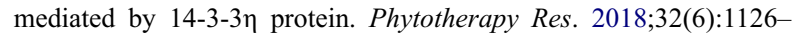
1134. doi: $10.1002 /$ ptr.6053

19. Li X, Wu C, Chen N, et al. PI3K/Akt/mTOR signaling pathway and targeted therapy for glioblastoma. Oncotarget. 2016;7(22):33440.

20. Liu P, Zhao H, Wang R, et al. MicroRNA-424 protects against focal cerebral ischemia and reperfusion injury in mice by suppressing oxidative stress. Stroke. 2015;46(2):513-519. doi:10.1161/STROKEAHA. 114.007482

21. Ferrara N, Adamis AP. Ten years of anti-vascular endothelial growth factor therapy. Nat Rev Drug Discov. 2016;15(6):385.

22. Bahr Hosseini M, Hou J, Bikson M, Iacoboni M, Saver JL. Abstract TP72: transcranial Direct Current Stimulation (tDCS) for neuroprotection in acute cerebral ischemia: meta-analysis of preclinical studies and implications for human clinical trials. Stroke. 2018;49(Suppl_1): ATP72-ATP72. doi:10.1161/str.49.suppl 1.TP72

23. Enzmann G, Kargaran S, Engelhardt B. Ischemia-reperfusion injury in stroke: impact of the brain barriers and brain immune privilege on neutrophil function. Ther Adv Neurol Disord. 2018;11:17562864187 94184. doi:10.1177/1756286418794184
24. Zhan J, Xiaoqiong L, Hao R. Effect of dendrobium nobile polysaccharides on focal cerebral ischemia/reperfusion rats. Chin $J$ Cerebrovasc Dis. 2017;14(1):25-31.

25. Huang J-L, Liu -W-W, Sun X-J. Hydrogen inhalation improves mouse neurological outcomes after cerebral ischemia/reperfusion independent of anti-necroptosis. Med Gas Res. 2018;8(1):1. doi:10.4103/2045-9912.229596

26. Xie W, Zhou P, Sun Y, et al. Protective effects and target network analysis of ginsenoside Rg1 in cerebral ischemia and reperfusion injury: a comprehensive overview of experimental studies. Cells. 2018;7(12):270. doi:10.3390/cells7120270

27. Zheng Q, Wang B, Gao J, et al. CD 155 knockdown promotes apoptosis via AKT/Bcl-2/Bax in colon cancer cells. J Cell Mol Med. 2018;22(1):131-140. doi:10.1111/jcmm.13301

28. Rogers C, Erkes DA, Nardone A, Aplin AE, Fernandes-Alnemri T, Alnemri ES. Gasdermin pores permeabilize mitochondria to augment caspase-3 activation during apoptosis and inflammasome activation. Nat Commun. 2019;10(1):1689. doi:10.1038/s41467-019-09397-2

29. Sinha D, Kalimutho M, Bowles J, et al. Cep55 overexpression causes male-specific sterility in mice by suppressing Foxo1 nuclear retention through sustained activation of PI3K/Akt signaling. Faseb J. 2018;32 (9):4984-4999. doi:10.1096/fj.201701096RR

30. Sun D, Huang J, Zhang Z, et al. Luteolin limits infarct size and improves cardiac function after myocardium ischemia/reperfusion injury in diabetic rats. PLoS One. 2012;7(3):e33491. doi:10.1371/ journal.pone.0033491

31. Bavarsad K, Barreto GE, Sahebkar A. Protective effects of curcumin against ischemia-reperfusion injury in the nervous system. Mol Neurobiol. 2019;56(2):1391-1404. doi:10.1007/s12035-018-1169-7

32. Conrad M, Kagan VE, Bayir H, et al. Regulation of lipid peroxidation and ferroptosis in diverse species. Genes Dev. 2018;32(910):602-619. doi:10.1101/gad.314674.118

33. Ighodaro O, Akinloye O. First line defence antioxidants-superoxide dismutase (SOD), catalase (CAT) and glutathione peroxidase (GPX): their fundamental role in the entire antioxidant defence grid. Alexandria J Med. 2018;54(4):287-293. doi:10.1016/j.ajme.2017. 09.001

34. Shekhar S, Jain S, Priya P. Assessment of serum antioxidant enzymes superoxide dismutase (SOD) and glutathione peroxidase in oral submucous fibrosis. J Adv Med Dent Sci Res. 2019;7(1):1-5.

35. Chen C, Li T, Zhao Y, et al. Platelet glycoprotein receptor Ib blockade ameliorates experimental cerebral ischemia-reperfusion injury by strengthening the blood-brain barrier function and anti-thromboinflammatory property. Brain Behav Immun. 2018;69:255-263. doi:10.1016/j.bbi.2017.11.019

36. Sun JC, Wua ST, Lee KJ, Sun YM. Docking prediction for luteolin

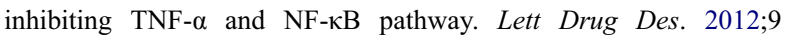
(2):169-176. doi:10.2174/157018012799079707
Neuropsychiatric Disease and Treatment

\section{Publish your work in this journal}

Neuropsychiatric Disease and Treatment is an international, peerreviewed journal of clinical therapeutics and pharmacology focusing on concise rapid reporting of clinical or pre-clinical studies on a range of neuropsychiatric and neurological disorders. This journal is indexed on PubMed Central, the 'PsycINFO' database and CAS, and is the official journal of The International Neuropsychiatric Association (INA). The manuscript management system is completely online and includes a very quick and fair peer-review system, which is all easy to use. Visit http://www.dovepress.com/testimonials.php to read real quotes from published authors. 